\title{
AN ADAPTIVE FAST NONLINEAR ANALYSIS (AFNA) ALGORITHM FOR RAPID TIME HISTORY ANALYSIS
}

\author{
Bowei Li ${ }^{1}$, Wei-Chu Chuang ${ }^{1}$, and Seymour M.J. Spence ${ }^{1}$ \\ ${ }^{1}$ Department of Civil and Environmental Engineering, University of Michigan \\ 2350 Hayward St, Ann Arbor, MI, USA \\ e-mail: \{jacklbw,wechuang,smjs\}@umich.edu
}

\begin{abstract}
Performance-based earthquake engineering is becoming ever-more popular due to its capability to deliver structures that rationally meet society's need for a safe built environment. An important barrier to the application of this approach is the high computational demand required for providing detailed and accurate response characterization through nonlinear time history analysis. In this paper, an efficient adaptive fast nonlinear analysis (AFNA) algorithm is proposed to address this issue. This novel step-by-step integration scheme adaptively updates all solver configurations in real-time, including number of pseudo modes considered in the analysis, time step size, and potential nonlinear elements, in order to efficiently evaluate the nonlinear dynamic responses. In particular, in modeling potential inelasticity, a distributed plasticity model is considered to allow inelasticity to distribute along the length and height of the elements. The practicality and potential of the proposed algorithm are illustrated on a full scale archetype building subject to stochastic seismic excitations. The proposed algorithm is shown to be around an order of magnitude faster than traditional direct integration schemes while achieving a remarkable accuracy in both global response time histories and local hysteretic responses, therefore enabling efficient application of probabilistic performance-based design frameworks that often require repeated evaluation of nonlinear dynamic systems.
\end{abstract}

Keywords: Performance-based Earthquake Engineering, Inelastic Systems, Structural Dynamics. 


\section{INTRODUCTION}

Performance-based earthquake engineering (PBEE) has gained immense interest in the past decades $[1,2,3,4,5]$, and has recently been extended to wind engineering $[6,7,8,9,10,11$, $12,13,14]$. The popularity of PBEE can be traced back to its ability to meet society's desire for a safe built environment at reasonable economic cost. One of the key difficulties in the applications of PBEE in practice is the potentially significant computational demand associated with evaluating nonlinear responses of structural systems subject to extreme seismic events through nonlinear analysis. This computational demand can become intractable in the case of probabilistic PBEE based on stochastic simulation as such approaches generally require the repeated evaluation of the system. The most straightforward solution for this issue is to improve analysis efficiency by replacing traditional time consuming nonlinear time history analysis with computationally tractable alternatives. While approaches such as pushover analysis can meet the efficiency requirement, these approaches are generally incapable of capturing cumulative damage mechanisms or effects of higher modes $[15,16,11]$, nor are they able to provide full time history responses.

Fast nonlinear analysis (FNA) [17] is a promising nonlinear time history analysis approach to overcome the aforementioned limitations. This approach keeps the concept of modal coordinate transformation and truncation, as in linear elastic analysis, which not only allows the equations of motion to be partially decoupled, such that more accurate and efficient piece-wise exact solutions can be used, but also enables the possibility to exclude unnecessary modes to lower the dimensionality of the problem for better efficiency. Based on a pre-designated set of elements that are expected to be nonlinear, this approach only considers these elements during the solution process, and thus enjoys significant gains in computational efficiency. However, the need to pre-designate elements that will experience inelasticity greatly reduces the general applicability of the FNA approach. In this work, an adaptive FNA (AFNA) is developed to address this limitation, while preserving almost all the advantages of the FNA approach. The resulting approach provides remarkably accurate full time history results at a fraction of the computational demand required by state-of-the-art direct integration schemes. The proposed approach adjusts the solver configurations, e.g. number of pseudo modes, time step size, and potential nonlinear elements, in real-time based on the state of the system, such that unnecessary computations and the need of a pre-designated set of potential nonlinear elements are eliminated. In modeling inelasticity, a distributed plasticity model is considered in this work, allowing inelasticity to distribute along the length and depth of the elements. A full scale archetype building subject to stochastic seismic excitations is presented to illustrate the efficiency and potential of the proposed approach.

\section{PROBLEM SETUP}

A structural system subject to dynamic load effects can in general be modeled as a multidegree-of-freedom (MDOF) system subject to stochastic excitations:

$$
\boldsymbol{M} \ddot{\boldsymbol{u}}(t)+\boldsymbol{C} \dot{\boldsymbol{u}}(t)+\boldsymbol{f}_{\mathrm{nl}}(t)=\boldsymbol{F}(t)
$$

where $\boldsymbol{M}, \boldsymbol{C}$ are respectively the structural mass and damping matrices; $\boldsymbol{u}(t)$ is the displacement vector while $\dot{\boldsymbol{u}}(t)$ and $\ddot{\boldsymbol{u}}(t)$ are its first and second derivatives with respect to time, i.e. the velocity and acceleration vectors; $\boldsymbol{F}(t)$ is the external force vector while, considering a fiber-based discretization, $\boldsymbol{f}_{\mathrm{nl}}(t)$ is the following nonlinear force vector:

$$
\boldsymbol{f}_{\mathrm{nl}}(t)=\boldsymbol{B}^{\mathrm{T}} \boldsymbol{W} \boldsymbol{\sigma}(t)
$$


where $\boldsymbol{B}$ is the compatibility matrix transforming responses at global degree of freedoms (DOF) to fiber responses; $\boldsymbol{W}$ is a diagonal matrix collecting multiplication factors to perform numerical integration for each of the fiber stress, typically obtained from the integration weights, fiber areas, as well as element lengths; and $\boldsymbol{\sigma}(t)$ is the vector that collects the fiber stress at all integration points of finite element discretization.

\section{The adaptive fast nonlinear analysis method}

In this section, the AFNA method is developed by making the solver configurations, including time step size, number of pseudo modes, and set of potential nonlinear elements, of the FNA approach adaptive in time. This eliminates the requirement of a pre-designated set of potential nonlinear elements. Methods are outlined to this end that do not compromise the original merits of the FNA approach.

\subsection{The fast nonlinear analysis method}

By moving the nonlinear force vector $\boldsymbol{f}_{\mathrm{nl}}(t)$ to the right hand side, and introducing an pseudo positive definite stiffness matrix $\boldsymbol{K}_{\mathrm{e}}$ to prevent singularity, Equation (1) can be rewritten as:

$$
\boldsymbol{M} \ddot{\boldsymbol{u}}(t)+\boldsymbol{C} \dot{\boldsymbol{u}}(t)+\boldsymbol{K}_{\mathrm{e}} \boldsymbol{u}(t)=\boldsymbol{F}(t)+\boldsymbol{K}_{\mathrm{e}} \boldsymbol{u}(t)-\boldsymbol{f}_{\mathrm{nl}}(t)
$$

It should be noted that, despite how the pseudo stiffness matrix $\boldsymbol{K}_{\mathrm{e}}$ can be any arbitrary positive definite matrix, it is recommended to be taken as the initial elastic stiffness matrix of the structural system. The P-Delta effect can be further taken into account by considering a reduced stiffness matrix $[17,18]$.

By denoting $\boldsymbol{f}_{\text {nlc }}(t)=\boldsymbol{K}_{\mathrm{e}} \boldsymbol{u}(t)-\boldsymbol{f}_{\mathrm{nl}}(t)$ as the nonlinear correction force, Equation (3) can be decoupled on the left hand side using the modal approach, as follows:

$$
\Theta \ddot{\boldsymbol{q}}(t)+\boldsymbol{\Lambda} \dot{\boldsymbol{q}}(t)+\boldsymbol{\Omega} \boldsymbol{q}(t)=\hat{\boldsymbol{F}}(t)+\hat{\boldsymbol{f}}_{\mathrm{nlc}}(t)
$$

where $\boldsymbol{q}(t)$ is the modal displacements while $\dot{\boldsymbol{q}}(t)$ and $\ddot{\boldsymbol{q}}(t)$ are its first and second derivatives with respect to time; $\Theta=\Phi^{\mathrm{T}} \boldsymbol{M} \boldsymbol{\Phi}, \Lambda=\Phi^{\mathrm{T}} C \Phi$, and $\Omega=\boldsymbol{\Phi}^{\mathrm{T}} K_{\mathrm{e}} \boldsymbol{\Phi}$ are respectively the diagonal generalized mass, damping and stiffness matrices; $\Phi$ is the pseudo mode matrix consisting of the generalized eigenvectors of $\boldsymbol{M}$ and $\boldsymbol{K}_{\mathrm{e}}$. It is worth noting that the generalized masses representing inertial effects can be non-zero or zero, which correspond to dynamic or static modes respectively. In practice, higher order dynamic modes can be considered as static modes by ignoring their inertial effects in order to enhance both the efficiency and stability of dynamic solvers. In Equation (3), $\hat{\boldsymbol{F}}(t)=\boldsymbol{\Phi}^{\mathrm{T}} \boldsymbol{F}(t)$ is the generalized force vector; $\hat{\boldsymbol{f}}_{\text {nlc }}(t)=\boldsymbol{\Phi}^{\mathrm{T}} \boldsymbol{f}_{\text {nlc }}(t)$ is the generalized nonlinear correction force vector, which can be evaluated as:

$$
\hat{\boldsymbol{f}}_{\mathrm{nlc}}(t)=\boldsymbol{\Psi}^{\mathrm{T}} \boldsymbol{W}\left[\boldsymbol{\sigma}_{\mathrm{e}}(t)-\boldsymbol{\sigma}(t)\right]
$$

where $\Psi=\boldsymbol{B} \Phi$ is the modal strain matrix; $\boldsymbol{\sigma}_{\mathrm{e}}(t)$ is the fiber stress elastic predictor for all fibers evaluated with the elastic modulus and fiber strains $\epsilon(t)$ as:

$$
\boldsymbol{\epsilon}(t)=\boldsymbol{\Psi} \boldsymbol{q}(t)
$$

It can be noted that when a fiber stress is in the linear elastic range, it does not contribute to $\hat{\boldsymbol{f}}_{\text {nlc }}(t)$, and thus can be ignored. In practice, the FNA only considers a pre-designated set of potential nonlinear elements, such that Equation (5) can be evaluated with much higher efficiency if only a portion of the elements are potentially nonlinear. 
The FNA solves Equation (3) in a step by step manner, with a step size of $\Delta t$, and starts with an initial guess solution at each of the time step through the Taylor expansion:

$$
\begin{array}{r}
\boldsymbol{q}^{(0)}(t+\Delta t)=\boldsymbol{q}(t)+\Delta t \dot{\boldsymbol{q}}(t)+\frac{\Delta t^{2}}{2} \ddot{\boldsymbol{q}}(t) \\
\dot{\boldsymbol{q}}^{(0)}(t+\Delta t)=\dot{\boldsymbol{q}}(t)+\Delta t \ddot{\boldsymbol{q}}(t)
\end{array}
$$

where the superscripts on the left hand side indicates the number of iterations. At the $k$ th iteration, $\boldsymbol{q}^{(k)}(t+\Delta t)$ and $\dot{\boldsymbol{q}}^{(k)}(t+\Delta t)$ will be used to determine fiber strains and subsequently fiber stresses $\boldsymbol{\sigma}_{\mathrm{e}}(t)$ and $\boldsymbol{\sigma}(t)$ through the fiber material constitutive law. Finally, $\hat{\boldsymbol{f}}_{\text {nlc }}^{(k)}(t)$ can be estimated through integration. The solution at the next iteration, i.e. $\boldsymbol{q}^{(k+1)}(t+\Delta t)$ and $\dot{\boldsymbol{q}}^{(k+1)}(t+\Delta t)$, is obtained by the piece-wise exact solution [19] with the external loads considered as $\hat{\boldsymbol{F}}(t)+\hat{\boldsymbol{f}}_{\text {nlc }}^{(k)}(t)$. This iterative process continues until convergence is reached. The algorithm performs the process described above for each time step over the time range of interest to provide response time histories.

\subsection{The adaptive solution procedure}

The main limitation of the FNA approach is the requirement of a pre-designated set of potential nonlinear elements. The AFNA presented in this section addresses this issue by determining the potential nonlinear elements during analyses in real-time. This feature allows AFNA to detect the existence of nonlinear elements, and further enables AFNA to shift between linear elastic and nonlinear solvers with different time steps and number of pseudo modes.

In the implementation, modes with natural frequencies greater than a threshold of interest, $f_{\text {st }}$, will be considered as static by ignoring their generalized mass or inertial effect, while the reaming modes are considered as dynamic. In solving the response time histories, the algorithm first assumes that the structural system is linear elastic and moves ahead with a relatively large time step $\Delta^{\mathrm{L}} t$ while considering only the dynamic modes. At the end of each time step $\Delta^{\mathrm{L}} t$, fiber strains and stresses are determined based on the estimated solutions $\boldsymbol{q}\left(t+\Delta^{\mathrm{L}} t\right)$ and $\dot{\boldsymbol{q}}(t+$ $\left.\Delta^{\mathrm{L}} t\right)$ for the assumed linear elastic step. The algorithm will proceed to the next time step if all fibers remain in their linear elastic loading/unloading/reloading range. Otherwise, the algorithm moves back to the previous time step $t$ and solves until reaching the time $t+\Delta^{\mathrm{L}} t$ using a smaller time step $\Delta^{\mathrm{S}} t=\Delta^{\mathrm{L}} t / \eta$, in which $\eta$ is an integer such that the $\Delta^{\mathrm{S}} t$ is not greater than half the natural period of the highest dynamic mode $T_{\min }$, i.e. $\Delta^{\mathrm{S}} t \leq T_{\min } / 2$, to ensure stability of the approach. In general, the choice of eta $=4$ will provide a good balance between accuracy and efficiency. Within each small time step $\Delta^{\mathrm{s}} t$ the algorithm first forms a list of potential nonlinear elements by checking the fiber strains or stresses based on the predicted solutions of Equation (7). The solution process then enters the iterative scheme as in the FNA with all modes considered, if the list of potential nonlinear elements is not empty. Otherwise, it proceeds to the next small time step using the piece-wise exact solution considering the dynamic modes of the larger time step phase without iteration. Within this context, when the algorithm reaches $t+\Delta^{\mathrm{L}} t$, the solution process then proceeds to the next time step considering the initial step size $\Delta^{\mathrm{L}} t$ and starts another adaptive process if necessary. The entire process is then terminated when reaching the end of the time sequence, providing a full range of inelastic response histories for structures subject to dynamic external loads. The overall solution process is illustrated in the flow chart of Figure 1. 


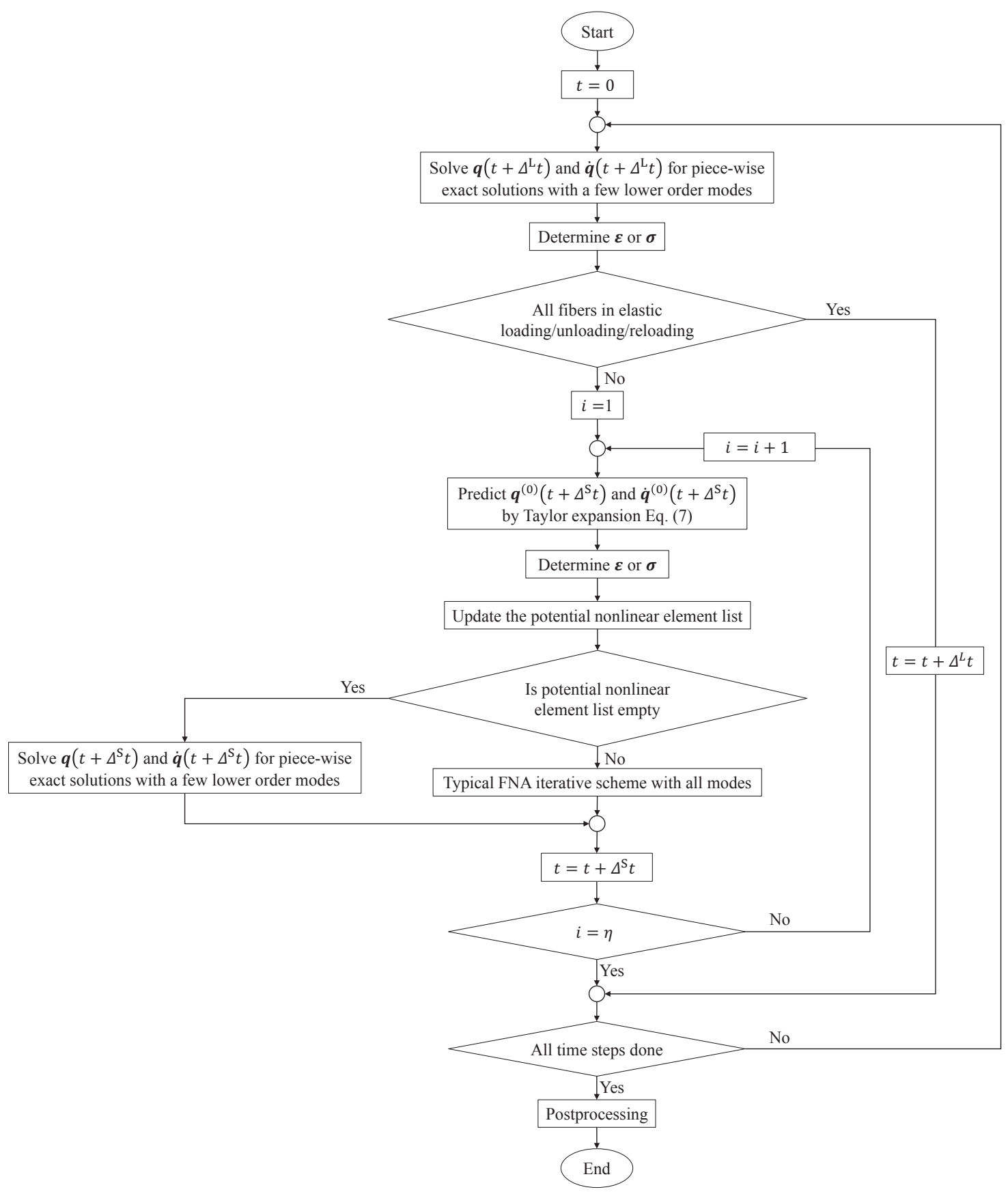

Figure 1: Flowchart illustrating the steps of the AFNA algorithm. 


\section{CASE STUDY}

A 37-story 6 span steel moment resisting frame assumed to be located in downtown San Francisco is considered in this case study. The width of each span is $5 \mathrm{~m}$ while the story height is $6 \mathrm{~m}$ for the first floor and $4 \mathrm{~m}$ for the remaining floors, leading to a total height of $150 \mathrm{~m}$. Box sections and W24 sections are considered respectively for columns and beams, with section sizes summarized in Table 1. The elastic modulus and the yield stress are respectively taken as $E_{s}=200 \mathrm{GPa}$ and $\sigma_{y}=355 \mathrm{MPa}$. The structural mass is modeled to be lumped at each floor considering combined self and carried mass of $100 \mathrm{~kg} / \mathrm{m}^{3}$, and super dead load of $23.5 \mathrm{kN} / \mathrm{m}$.

\begin{tabular}{ccc}
\hline Floor & Beams & Columns* \\
\hline $1-20$ & W $24 \times 192$ & $50^{2} \times 2.5$ \\
$21-30$ & W $24 \times 103$ & $40^{2} \times 2.0$ \\
$31-40$ & W $24 \times 103$ & $35^{2} \times 1.8$ \\
\hline *(outer side size $)^{2} \times($ wall thickness $)$
\end{tabular}

Table 1: Sections with dimensions in $(\mathrm{cm})$.
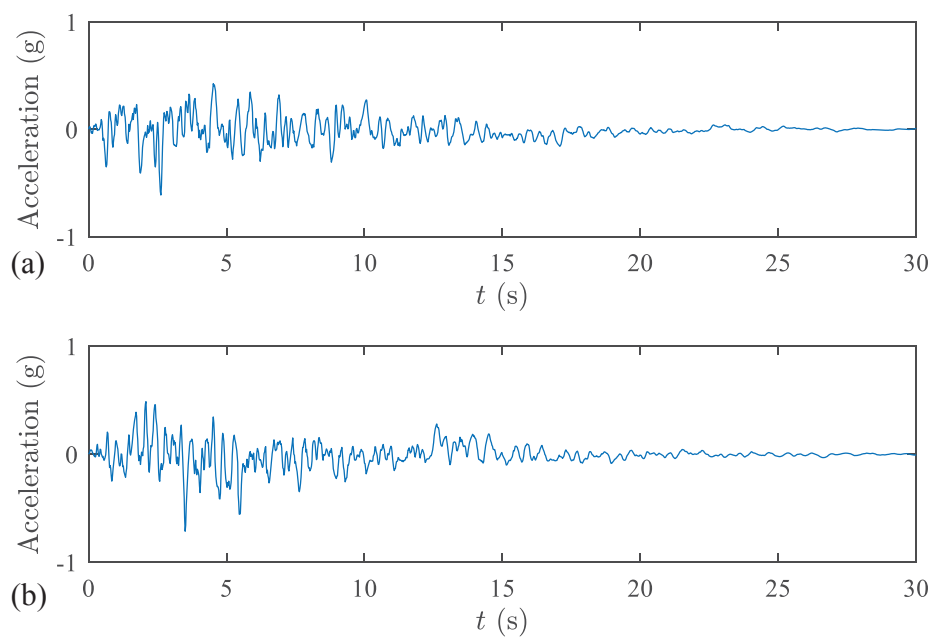

Figure 2: The two synthetic ground motion samples used in the case study: (a) GM1 (b) GM2.

The structure is assumed to be subjected to a $10 \%$ exceedance probability in 50-year seismic hazard. Synthetic ground motions are generated via the model proposed by Rezaeian and Der kiureghian [20], with the filter frequency and damping optimized to fit the target spectrum constructed from the USGS unified hazard tool [21] for the building site, with the rest of the parameters chosen to be consistent with Loma Prieta records (Moment magnitude $=6.93$, Rupture distance $=18.3 \mathrm{~km})$. The two representative synthetic ground motion $(\mathrm{GM})$ samples used in this work are shown in Figure 2. For comparison, nonlinear time history analyses based on the GM samples was carried out by direct integration in OpenSees. Elements experiencing inelasticity subject to the two GM samples are marked in red in Figure 3. It can be seen that for the two cases (GM1 and GM2) the structure experiences, respectively, moderate and significant nonlinearity. 

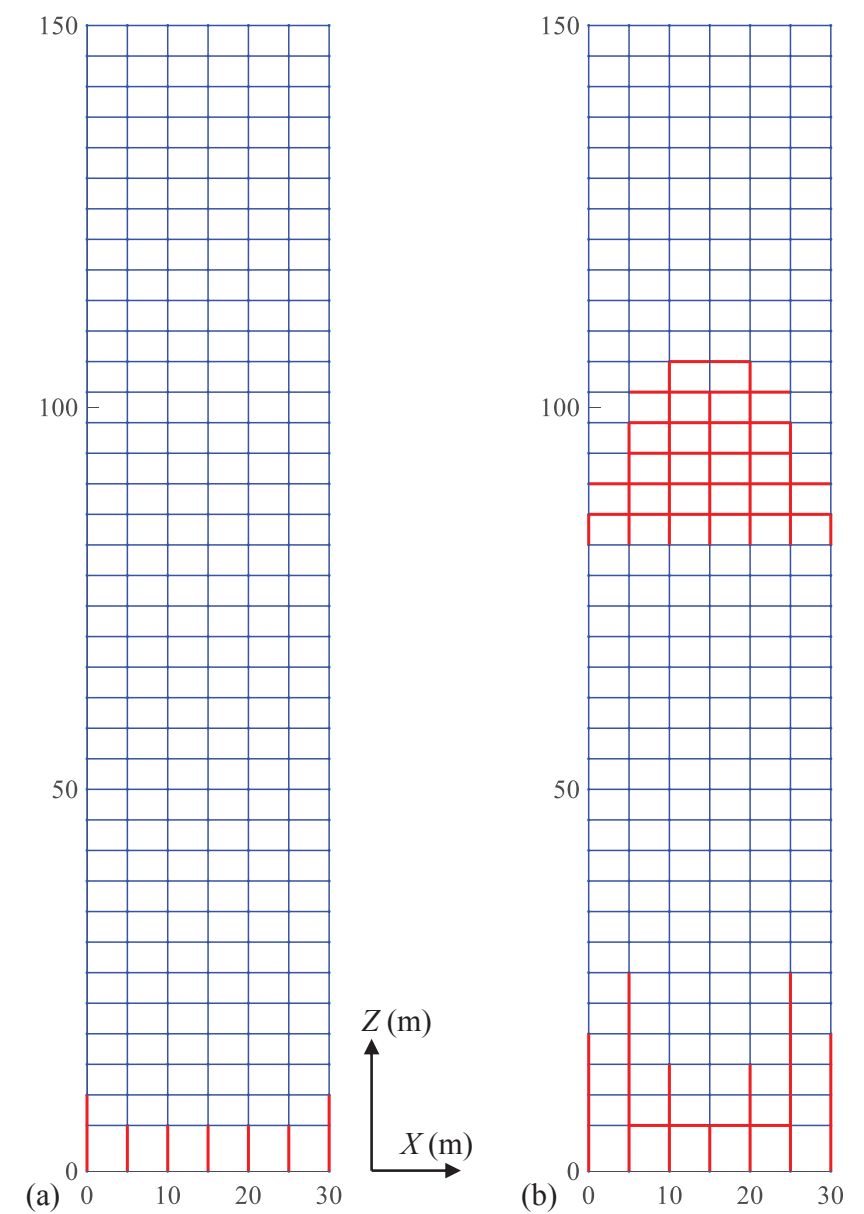

Figure 3: Elements experiencing nonlinearity (in red): (a) GM1 (b) GM2.

All results were estimated using a machine with Intel(R) Core(TM) i7-8750H CPU @ 2.20GHz processor and 16 GB RAM. The comparison in terms of computational times is reported in Table 2. It is seen that the AFNA is around an order of magnitude faster than the state-of-the-art nonlinear analysis approach used in OpenSees for GM1, i.e. for moderate nonlinearity. Despite how a lower gain in efficiency is achieved for GM2 (significant nonlinearity), the AFNA is still notably faster. It should also be noted that the AFNA approach was programmed in Matlab and, unlike the OpenSees solvers, was not fully optimized for computational efficiency. Thus, based on the results, the AFNA algorithm is expected to be around an order of magnitude faster than the direct integration even in the case of significant nonlinearity.

The comparison between the displacements under GM1 and GM2 at the 10th, 20th, and 37 th floor obtained from direct integration and the AFNA approaches are reported in Figure 4. A perfect correspondence can be observed over the entire time history. In addition, Figure 5 reports the comparison between the fiber strain and stress time histories and hysteretic curves for an extreme fiber of the exterior first floor column for the two ground motions. It is seen that the AFNA approach still achieves remarkable accuracy, even for highly localized responses, e.g. nonlinear fiber strains or stresses, which are generally challenging to compute accurately and efficiently due to the potentially large number of modes involved. Lastly, it is worth noting that, despite how an elastic perfectly plastic (EPP) material was considered in the case study, 

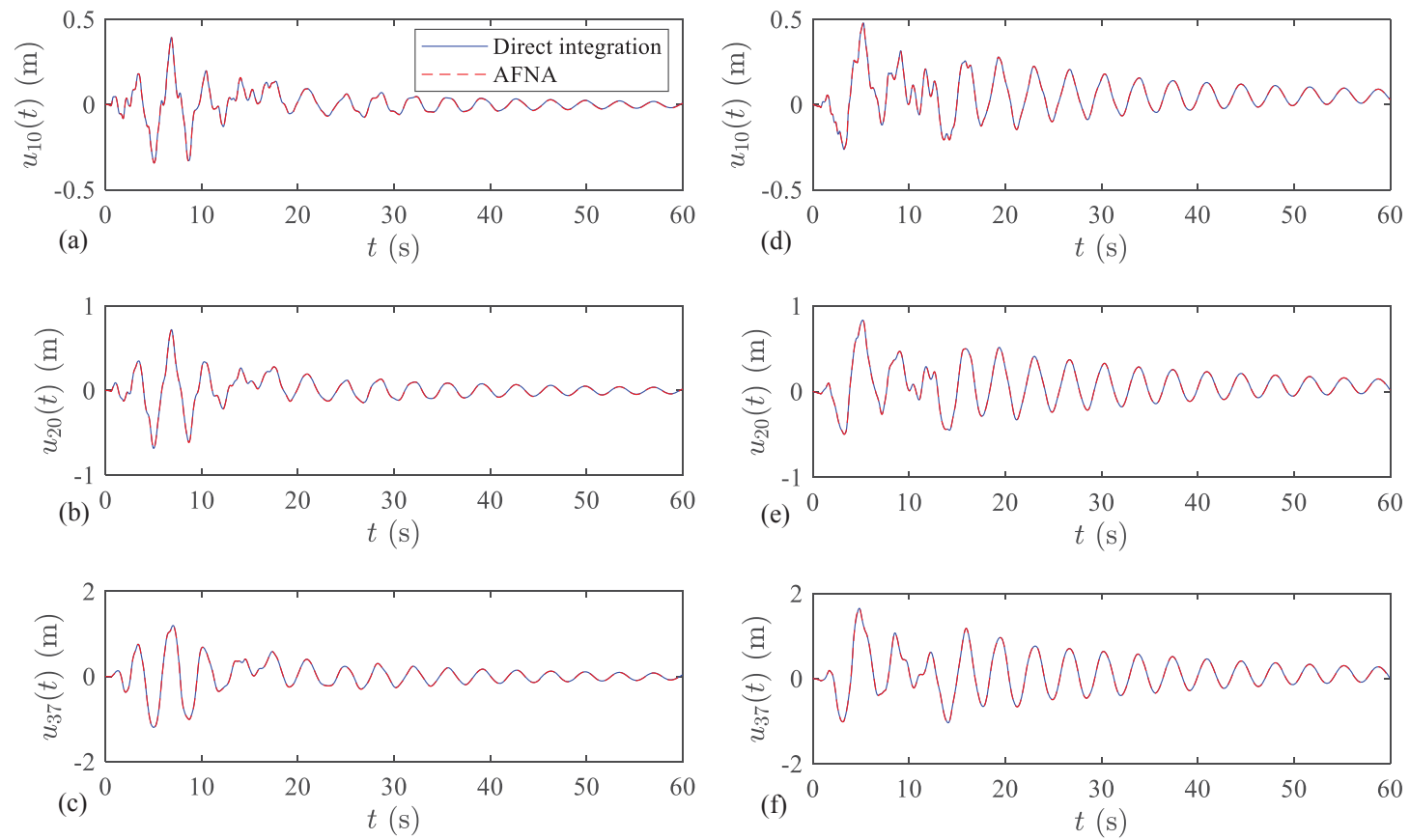

Figure 4: Comparison of displacements obtained by direct integration and AFNA for: GM1 at (a) the 10th floor, (b) the 20th floor (c), the 37th floor; and for GM2 at (d) the 10th floor, (e) the 20th floor, (f) and the 37th floor.
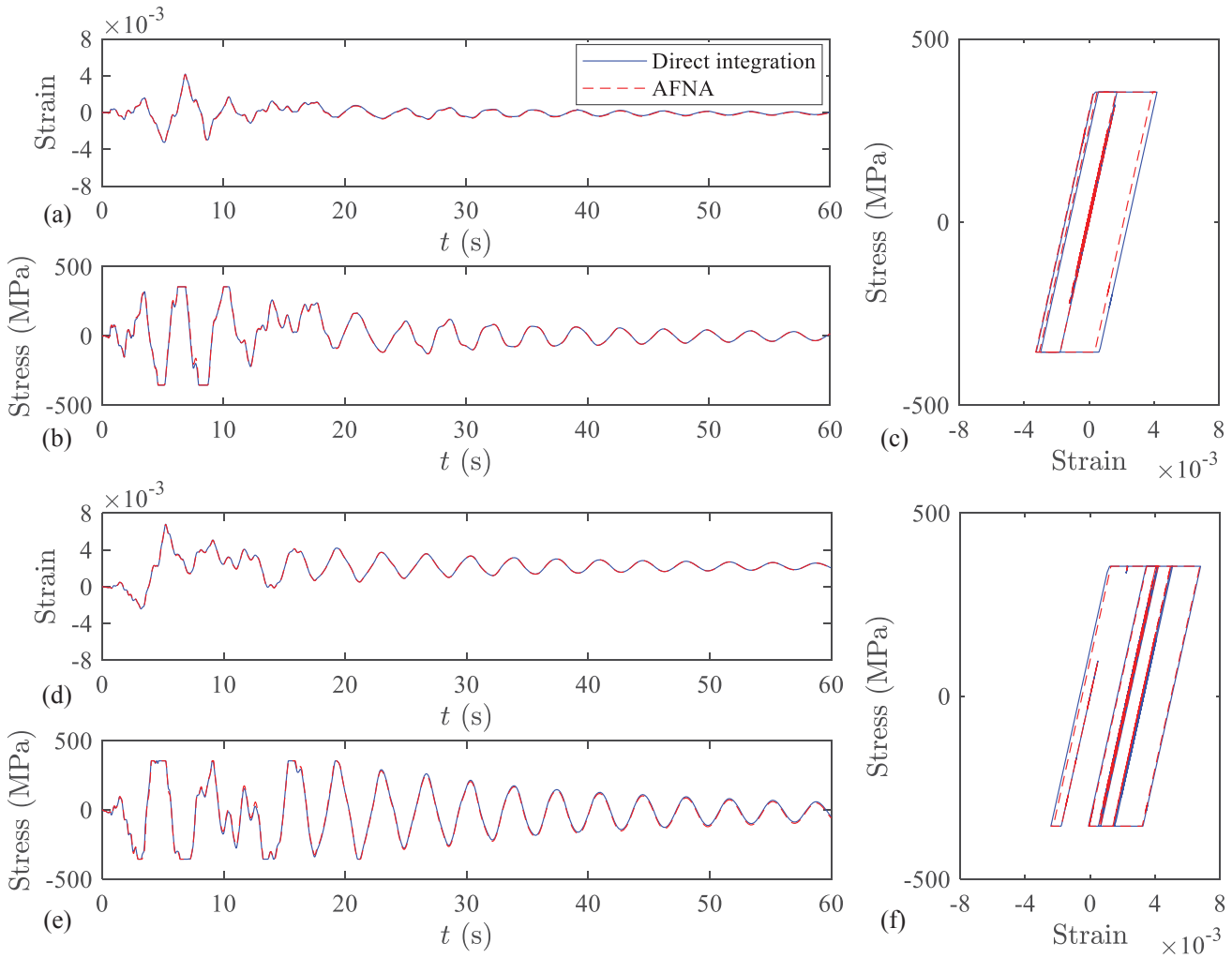

Figure 5: Extreme fiber responses for the exterior first floor column. Comparison between direct integration and AFNA: (a) strain time history for GM1; (b) stress time history for GM1; (c) hysteretic curve for GM1; (d) strain time history for GM2; (e) stress time history for GM2; (f) hysteretic curve for GM2. 


\begin{tabular}{ccc}
\hline Ground motions & GM1 & GM2 \\
\hline Computational time: Direct integration (s) & 64.58 & 63.90 \\
Computational time: AFNA (s) & 7.03 & 15.10 \\
\hline
\end{tabular}

Table 2: Computational time for the nonlinear time history analyses.

the proposed algorithm can be used for materials with general constitutive relations.

\section{CONCLUSION}

In this work, a highly efficient adaptive fast nonlinear analysis (AFNA) algorithm was developed, as a computational efficient alternative to the direct integration approach to provide full time history responses for computationally intensive engineering applications, e.g. performancebased earthquake engineering. The algorithm implements the modal analysis concept that partially decouples the equation of motions, such that piece-wise linear solutions are applicable. Meanwhile, the algorithm updates the potential nonlinear elements, time step size, as well as the number of pseudo modes to be considered in real-time during the solution process. In particular, in defining the structural model, fiber-based distributed plasticity models were considered. The results show that the AFNA algorithm is capable of accurately estimating both the global response time histories and local hysteretic behaviors with around an order of magnitude less computational time as compered to state-of-the-art direct integration approaches. Finally, it is worth noting that even though only seismic input was considered in the case study, the proposed algorithm can also be directly applied to cases subject to stochastic wind loads. In this case, the algorithm is expected to provide even greater time savings, as typically less nonlinearity is experienced by structures subject to winds. Overall, the notable efficiency and accuracy of the proposed AFNA approach illustrates its potential in computationally intensive applications, such as probabilistic performance-based design frameworks, that require repeated evaluation of nonlinear dynamic systems.

\section{ACKNOWLEDGEMENT}

This research effort was supported in part by the Magnusson Klemencic Associates (MKA) Foundation through Research Grant Agreement No. 101 and the National Science Foundation through grant number CMMI-1750339. This support is gratefully acknowledged.

\section{REFERENCES}

[1] H. Krawinkler. Challenges and progress in performance-based earthquake engineering. In International Seminar on Seismic Engineering for Tomorrow-In Honor of Professor Hiroshi Akiyama, volume 26, 1999.

[2] K.A. Porter. An overview of peer's performance-based earthquake engineering methodology. In Proceedings of ninth international conference on applications of statistics and probability in civil engineering, pages 1-8, 2003.

[3] J. Moehle and G.G. Deierlein. A framework methodology for performance-based earthquake engineering. In 13th world conference on earthquake engineering, volume 679, 2004. 
[4] S. Günay and K.M. Mosalam. Peer performance-based earthquake engineering methodology, revisited. Journal of Earthquake Engineering, 17(6):829-858, 2013.

[5] Federal Emergency Management Agency (FEMA). Seismic performance assessment of buildings volume 1-methodology. Rep. No. FEMA P-58-1, 2012.

[6] M. Ciampoli, F. Petrini, and G. Augusti. Performance-based wind engineering: towards a general procedure. Structural Safety, 33(6):367-378, 2011.

[7] S.M.J. Spence and A. Kareem. Performance-based design and optimization of uncertain wind-excited dynamic building systems. Engineering Structures, 78:133-144, 2014.

[8] S.M.J. Spence, E. Bernardini, and A. Kareem. A first step towards a general methodology for the performance-based design of wind-excited structures. In Structures Congress 2015, pages 1482-1493, 2015.

[9] S.M.J. Spence, W.C. Chuang, P. Tabbuso, E. Bernardini, A. Kareem, L. Palizzolo, and A. Pirrotta. Performance-based engineering of wind-excited structures: A general methodology. In Geotechnical and Structural Engineering Congress 2016, pages 1269-1282, 2016.

[10] W.C. Chuang and S.M.J. Spence. A performance-based design framework for the integrated collapse and non-collapse assessment of wind excited buildings. Engineering Structures, 150:746-758, 2017.

[11] W.C. Chuang and S.M.J. Spence. An efficient framework for the inelastic performance assessment of structural systems subject to stochastic wind loads. Engineering Structures, 179:92-105, 2019.

[12] Z. Ouyang and S.M.J. Spence. A performance-based damage estimation framework for the building envelope of wind-excited engineered structures. Journal of Wind Engineering and Industrial Aerodynamics, 186:139-154, 2019.

[13] A. Suksuwan and S.M.J. Spence. Performance-based design optimization of uncertain wind excited systems under system-level loss constraints. Structural Safety, 80:13-31, 2019.

[14] W.C. Chuang and S.M.J. Spence. Probabilistic performance assessment of inelastic wind excited structures within the setting of distributed plasticity. Structural Safety, 84:101923, 2020 .

[15] H. Krawinkler and G.D.P.K. Seneviratna. Pros and cons of a pushover analysis of seismic performance evaluation. Engineering structures, 20(4-6):452-464, 1998.

[16] S. Antoniou and R. Pinho. Advantages and limitations of adaptive and non-adaptive forcebased pushover procedures. Journal of Earthquake Engineering, 8(04):497-522, 2004.

[17] E.L. Wilson. Three-dimensional static and dynamic analysis of structures. 2002.

[18] E.L. Wilson and A. Habibullah. Static and dynamic analysis of multi-story buildings, including p-delta effects. Earthquake spectra, 3(2):289-298, 1987. 
[19] A.K. Chopra. Dynamics of Structures: Theory and Applications to Earthquake Engineering. Prentice-Hall, 2001.

[20] S. Rezaeian and A. Der Kiureghian. Simulation of synthetic ground motions for specified earthquake and site characteristics. Earthquake Engineering \& Structural Dynamics, 39(10):1155-1180, 2010.

[21] United States Geological Survey (USGS). Unified hazard tool, 2017. 